\title{
INTEGRAL EQUATION BASED METHOD FOR THE FAST ANALYSIS OF IRREGULARLY CONTOURED LARGE FINITE PHASED ARRAYS
}

\author{
Vakur B. Ertürk*, Özlem Aydın Çivi ${ }^{\dagger}$ \\ *Dept. of Electrical and Electronics Engineering, Bilkent University, Ankara, Turkey \\ e-mail: vakur@ee.bilkent.edu.tr \\ †Dept. of Electrical and Electronics Engineering, Middle East Technical University, Ankara, Turkey \\ e-mail: ozlem@metu.edu.tr
}

Keywords: Finite arrays; Method of Moments; Generalized Forward Backward Method.

\begin{abstract}
A fast and accurate integral equation based hybrid method that can investigate electrically large, arbitrarily contoured finite planar arrays of printed elements is developed. The method is a hybridization of the Galerkin type Method of Moments (MoM) and Generalized Forward Backward Method (GFBM) with the grounded dielectric slab's Green's function; and the acceleration of the resultant hybrid method by a discrete Fourier transform (DFT) based acceleration algorithm. Numerical results in the form of array current distribution are given for arbitrarily contoured as well as thinned arrays of probe fed microstrip patches where current on each element expanded by more than one subsectional basis function.
\end{abstract}

\section{Introduction}

Phased arrays of printed microstrip patches are vital in many commercial and military applications. Hence, their efficient and accurate analysis becomes crucial in order to obtain such arrays with better performance metrics. Unfortunately, majority of the developed analysis methods and available Computer Aided Design (CAD) tools are either slow or lack of rigor when they are used for the investigation of phased arrays of printed elements on grounded dielectric slabs in particular for the electrically large but finite and irregularly contoured ones. Moreover, if array thinning is required (so that the periodicity is deteriorated), solution process with the available tools becomes more demanding in terms of storage and computing time requirements.

In this study, we propose a fast and accurate integral equation based hybrid method that can investigate electrically large, arbitrarily contoured finite planar arrays of printed elements, which can be arbitrarily shaped probe-fed patches as well as printed dipoles [2]. The method is a hybridization of the Galerkin type Method of Moments (MoM) and Generalized Forward Backward Method (GFBM) [10] with the grounded dielectric slab's Green's function; and the acceleration of the resultant hybrid method by a discrete Fourier transform
(DFT) based acceleration algorithm [5]. The method also uses the "virtual element" concept [7] with "sub-arraying" so that arrays with irregular boundaries as well as sparse and nonperiodic arrays produced by array thinning process can be investigated without a major difficulty.

The solution process starts by introducing the virtual elements. Thus, regardless of the array shape, the array is extended to a two dimensionally periodic rectangular array. Then, an integral equation (IE) that uses the grounded dielectric slab's Green's function as its kernel is formed including the virtual elements and is solved with a combination of Galerkin type MoM and GFBM. Briefly, unknown current coefficients corresponding to a single element are initially solved by MoM. The solution for the unknown entire array current coefficients is then obtained through GFBM. Because the time-consuming matrix-vector products during the forward and backward sweeps of GFBM are the main bottle neck of the solution procedure, it is accelerated using a DFT based acceleration algorithm, which divides the array into strong and weak regions. However, as the complicated array elements have more than one basis functions per element, the available DFT based acceleration algorithm should be generalized. Hence, with the aid of the virtual elements, periodic sub-arrays are produced as many as the number of basis functions per element so that for each sub-array, one can use the available DFT based acceleration algorithm. The final result is the superposition of the subarray results.

It is worthwhile to mention at this point that effects of the virtual elements should be shed from the final solution as they are added to the actual array to maximize the efficiency of the solution procedure and to minimize the storage requirements of the method. Therefore, special conditions are imposed on them particularly during the strong region evaluations as explained in the subsection 2.3 .

Numerical results are provided for various irregularly contoured as well as thinned arrays using the proposed method and are compared with the results of a conventional hybrid MoM/Green's function method to assess the accuracy and efficiency of the proposed method. More examples will be provided during the presentation. 


\section{Formulation}

In this section, the formulation of the proposed method together with its salient features is provided starting by depicting a generic geometry of an irregularly contoured phased array. Then, we briefly discuss how GFBM is used to solve the MoM matrix equation obtained from the electric field integral equation (EFIE) that uses the Green's function for the grounded dielectric slab. Finally, implementation of the DFT based acceleration algorithm for irregularly contoured arrays is presented together with a discussion of the imposed conditions on virtual elements.

\subsection{Geometry}

Figure 1 illustrates a finite, arbitrarily contoured planar array of microstrip patches. The microstrip patches are printed on the dielectric-air interface $(z=0)$ of a grounded dielectric slab with a thickness $d$ and a relative dielectric constant $\varepsilon_{r}$. Each patch is assumed to be probe-fed with an ideal current source ( $\left.x_{\text {probe }} y_{\text {probe }}\right)$, has a length $L$, width $W$. In Figure 1 , actual elements of the array are depicted with blue (light-coloured for black and white illustrations) and they remain inside the dashed line that denotes the array boundary. Microstrip patches that lie outside the array boundary are denoted by red (dark-coloured) and they form the virtual elements. By including the virtual elements, a finite, periodic, rectangular array is obtained. In a similar fashion in the case of thinned arrays, the actual array may not be periodic. However, again by adding the virtual elements the thinned array is completed to a finite periodic rectangular array. Consequently, the final array (after the inclusion of virtual elements) to be solved with the proposed method is always a finite, periodic, planar array of $(2 N+1) \times(2 M+1)$ identical microstrip patches that are uniformly spaced from their neighbours by distances $d_{x}$ and $d_{y}$ in the $\hat{x}$ and $\hat{y}$ directions, respectively.

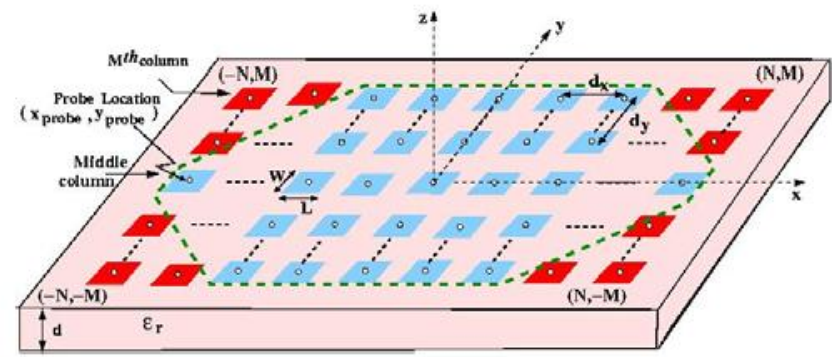

Figure 1. Geometry of a planar, irregularly contoured array of probe-fed microstrip patches.

\subsection{Method of Mom ents Formulation and the Generalized Forward Backward Method Solution for Phased Arrays}

Using an EFIE, whose kernel is the grounded dielectric slab Green's function [1], [3,4], [9], expanding the unknown induced array surface current in terms of a finite set of piecewise sinusoidal basis functions, and using a Galerkin procedure, a matrix-equation in the form of
\[ I=V \]
is formed. In (1), $I=\left[A_{n m r}\right]$ is the unknown column vector of current coefficients $A_{n m r}$ that represents the $r^{\text {th }}$ basis function belonging to the $n m^{\text {th }}$ patch, and $Z=\left\lfloor Z_{n m r, p q s}\right\rfloor$ is the impedance matrix of the array with entries $Z_{\eta m r, p q s}$ each of which denotes the mutual impedance between the $r^{\text {th }}$ basis function of the $\mathrm{nm}^{\text {th }}$ patch and $s^{\text {th }}$ testing function of the $p q^{\text {th }}$ patch. Finally, $V=\left\lfloor V_{p q s}\right\rfloor$ at the right hand side of (1) represents the known voltage vector responsible from the excitation of the array. Explicit expressions for $Z_{n m r, p q s}$ and $V_{p q s}$ can be found in $[1,2]$.

To solve (1) for the unknown current amplitudes, $A_{n m r}$, GFBM is used. Briefly, the current vector $I$ is decomposed into its forward, $I^{f}$, and backward, $I^{b}$, components. Similarly, the impedance matrix $Z$ is decomposed into three matrices, namely, $Z^{5 g}$ formed by the block diagonal matrices of $Z$ corresponding to the impedance matrix of a single patch, and $Z^{f B}$ and $Z^{b g}$ formed by the lower and upper triangular parts of $Z$, respectively, with $Z^{5 g}$ is subtracted. Then, the original matrix-equation given by (1) is expressed as

$$
\begin{aligned}
& \boldsymbol{Z}^{s g} I^{f}=V-\boldsymbol{Z}^{f g}\left(I^{f}+I^{b}\right) \\
& \boldsymbol{Z}^{s g} I^{f}=-\boldsymbol{Z}^{b g}\left(I^{f}+I^{b}\right)
\end{aligned}
$$

Initializing $I^{b}$ to zero at the first iteration, (2) is solved for $I^{f}$, and the resultant $I^{f}$ is used in (3) solve for $I^{b}$. To obtain the final converged result for the unknown current coefficients usually requires four or five iterations. Considering the fact that the total number of unknowns in (1) is $N_{\text {tot }}$, which is equal to the total number of antennas in the array multiplied by the total number of basis functions for each element, the computational complexity as well as the storage requirement of GFBM are $O\left(N_{\text {tot }}^{2}\right)$ due to the repeated and time-consuming computations of $Z^{f g} I$ and $Z^{b g} I$ in (2) and (3), respectively. Therefore, solving very large arrays using GFBM may not be feasible. However, with the DFT based acceleration algorithm proposed in [2], which is the generalized form of the algorithm explained in [5] and used in $[6,7,8]$, both the computational complexity and the storage requirements of the method reduces to $O\left(N_{\text {tot }}\right)$, which makes the method promising when dealing with very large arrays. A brief description of the method together with its salient features is provided in the following subsection.

\subsection{DFT Based Acceleration Algorithm}

For finite arrays, array current distribution is not uniform along the array and significant variations can be observed near the feed locations and at the array boundaries as shown in Figure 2(a), for a 19x19 element rectangular, probe-fed microstrip patch array (array and substrate parameters are given in the caption of the figure), and in Figure 3(a) for a 1501 element octagonal, probe-fed microstrip patch array 
(again array and substrate param eters are given in the caption of the figure). Furthermore, shape of the array boundary may also affect this current distribution as can be seen from the comparison of Figure 2(a) with Figure 3(a). However, the DFT spectrum of finite arrays are very compact as seen both in Figure 2(b) and Figure 3(b) regardless of how these arrays are contoured as long as they are relatively large. Therefore, working with the DFT spectrum of the array currents and selecting only the significant DFT terms usually provide very accurate results. The most important DFT term is the middle one (see both Figure 2(b) and Figure 3(b)), that corresponds to the DFT spectrum of an infinite array. Selecting this DFT term is a necessary condition and usually yields sufficient accuracy for many engineering applications. However, if more accuracy is desired, a few DFT terms around the middle DFT term can be taken.

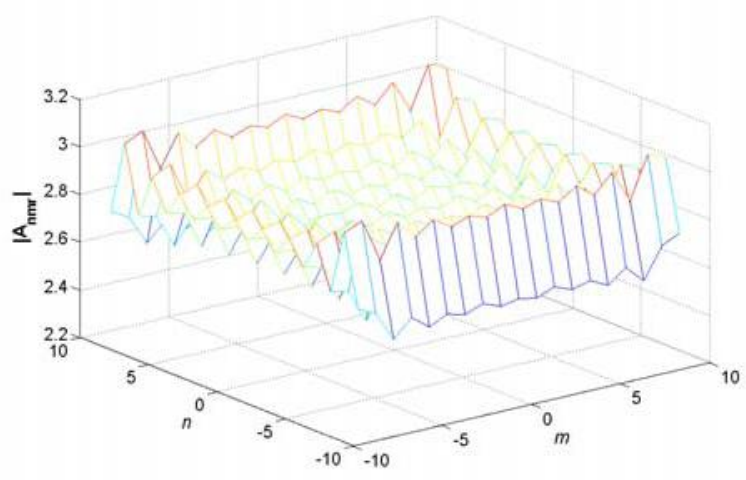

(a)

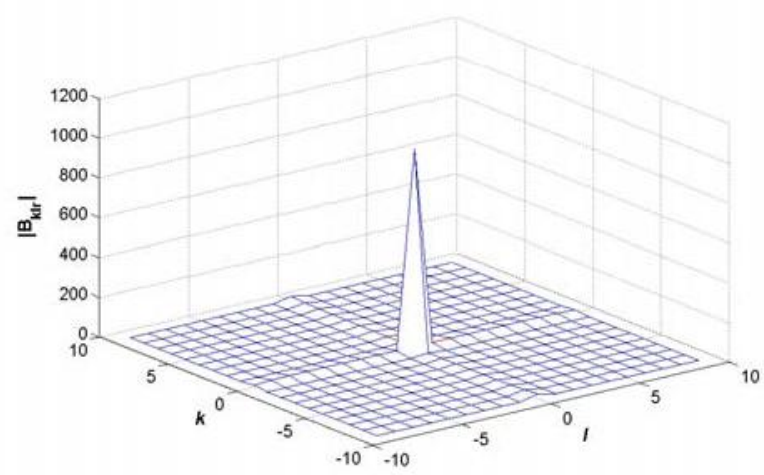

(b)

Figure 2. (a) Current amplitudes and (b) DFT spectrum of currents of $19 \times 19$ element rectangular probe-fed microstrip patch array. Array parameters are: $d=0.04 \lambda_{0}$, $\varepsilon_{\mathrm{r}}=2: 55, \mathrm{~L}=\mathrm{W}=0.3 \lambda_{0}, \mathrm{dx}=\mathrm{dy}=0.5 \lambda_{0}$, xprobe $=-\mathrm{L} / 2$, yprobe $=0$. Three $\hat{x}$-directed basis functions are used for each patch.

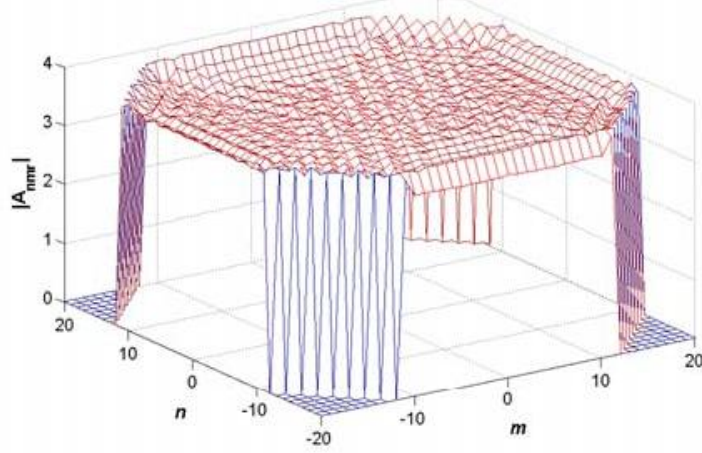

(a)

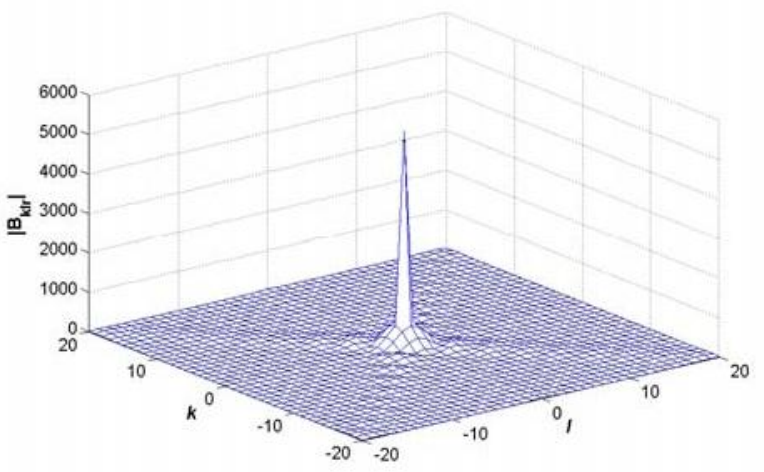

(b)

Figure 3. (a) Current amplitudes and (b) DFT spectrum of currents of 1501 element octagonal probe-fed microstrip patch array. Array parameters are: $d=0.04 \lambda_{0}, \varepsilon_{\mathrm{r}}=2: 55$, $\mathrm{L}=\mathrm{W}=0.3 \lambda_{0}, \mathrm{dx}=\mathrm{dy}=0.5 \lambda_{0}, \mathrm{xprobe}=-\mathrm{L} / 2, \mathrm{yprobe}=0$.

Three $\hat{x}$-directed basis functions are used for each patch.

The DFT based acceleration algorithm is based on this compactness of the DFT spectrum and very well suited for the fast and accurate solution of rectangular arrays. Therefore, as the first step of this algorithm, the array under study is mathematically extended to a rectangular array by introducing virtual elements. In the case of an irregularly contoured array, like the one depicted in Figure 1, virtual elements are added outside the boundary to make it a rectangular one. In the case of a thinned array, the array boundary might be a rectangular boundary. However, periodicity of the array might be distorted. Then, virtual elements must be added to make it a periodic array. As a result, the first step is to obtain a rectangular, periodic array of identical elements. Then, inside the GFBM, for both forward and backward sweeps, the DFT based acceleration algorithm $([2],[5,8])$ starts by dividing the contributing elements (elements that are in the front of a receiving element) into strong and weak interaction groups such that

$$
Z^{f g} I=\sum_{n, m, r \in s t r o n g} A_{n m r} Z_{n m r, p q s}+\sum_{n, m, r \in \text { weak }} A_{n m r} Z_{n m r, p q s}
$$


where strong and weak groups are denoted by strong and weak, respectively. The number of elements that form the strong group is fixed and very small compared to the entire array, but contributions coming from this group assure the fundamental accuracy of the overall solution. Thus, strong group contributions are obtained in an element-by-element fashion with the GFBM [2]. As mentioned above, inclusion of virtual elements helps us to obtain a periodic array from an irregularly contoured and/or from a thinned array so that both the computing time and storage requirements are minimized. However, effects of them should be shed from the final solution. Thus, in the computation of strong region contributions, voltages on these elements are set to zero (i.e., $V_{p q s}=0$ if $p q \in$ virtual elements) and mutual couplings related to these elements are set to zero (i.e., $Z_{n m r, p q s}=0$ if $n m$ and/or $p q \in$ virtual elements).

On the other hand, computation of the weak region contributions using GFBM without an acceleration algorithm constitutes the main bottleneck of the solution. However, these weak region contributions only provide minor corrections to the strong region contributions. Therefore, the DFT spectrum of the array current distribution is used in computing the weak region contributions by selecting only the significant DFT terms. It should be kept in mind that the current distribution on each element (probe-fed microstrip patch) is expanded using an arbitrary number of subsectional (piecewise sinusoidals) basis functions. Hence, the acceleration algorithm presented in [5] and used in $[6,8]$ is modified based on the two facts: (i) array elements are identical (i.e., each element is represented using the same number of basis functions), (ii) array is periodic. Briefly in the modified acceleration algorithm we first consider only the first basis function of each element and form a periodic subarray of elements represented by a single basis function. Then, the DFT acceleration algorithm used in $[6,8]$ is implemented. The same procedure is repeated for all other basis functions and all DFT contributions are superposed by a summation over the number of basis functions for a single patch $N_{b}$. As a result, the weak region contributions are represented by the generic equation given by

$$
[Z I]_{p q s ; w e a k}^{f g}=\sum_{r=1}^{N_{b}}\left[\sum_{k l \in Q} B_{k l r} C_{k l r, p q s}\right]
$$

where $\mathrm{Q}$ denotes the number of selected DFT terms, $B_{k d r}$ is the $k t^{\text {th }}$ DFT term of the subarray formed by the $r^{\text {th }}$ basis functions, and

$C_{k l r, p q s}=\sum_{n, m \in w e a k} Z_{n m r, p q s} e^{-j \beta_{x} n d_{x}} e^{-j \beta_{y} m d_{y}} e^{-j 2 \pi \frac{k n}{2 N+1}} e^{-j 2 \pi \frac{l m}{2 M+1}}$

represents the contribution of the $k l^{\text {th }}$ DFT term of the $r^{\text {th }}$ basis function to the $s^{\text {th }}$ basis function of the $p q^{\text {th }}$ receiving element.
Efficient evaluation of (6) is crucial for the efficiency of the method. Hence, (6) is computed in an iterative way as explained in [2].

One final remark is again how to shed the effects of virtual elements from the solution. As opposed to the strong region computations, during the computation of weak region contributions, all mutual coupling calculations are performed as if there is no virtual element in the entire array. The main reason is that in the computation of DFT coefficients (i.e., $\left.B_{k l r}\right), A_{n m r}$ values for virtual elements are zero. So, if all DFT terms are employed, utilization of nonzero coupling when a virtual element is involved does not affect the accuracy. In reality, we are using only a few (sometimes single) DFT terms that can yield a small error. Fortunately, such an error does not affect the overall accuracy of the solution.

Before providing the numerical results it should be mentioned that with the DFT based acceleration algorithm, the proposed method has an $O\left(N_{\text {tot }}\right)$ computational cost and storage requirements since both the number of significant DFT terms, $\mathrm{Q}$, and the size of the strong region are small compared to the total number of unknowns and they are fixed regardless of the size and shape of the array.

\section{Numerical Results and Discussion}

Numerical results in the form of array current distribution pertaining to various electrically large, irregularly contoured or thinned two-dimensional finite arrays of probe-fed microstrip patches on grounded dielectric slabs are given to assess the efficiency and accuracy of the proposed method.

The first numerical example is a finite array having an octagonal array boundary which contains 1501 patches. When the array is extended to a rectangular array by adding the virtual elements, the final array to be solved by the proposed method becomes a $41 \times 41$ array. All the patch, array and substrate related details are given in the caption of Figure 4, which shows the comparison for the magnitude of the array current coefficients, $\left|A_{n m r}\right|$ obtained with the proposed method, namely, DFT-GFBM and with the conventional hybrid MoM/Green's function technique for the $5^{\text {th }}$ column in Figure 4(a) and for the middle column in Figure 4(b). Use of 3 DFT terms with a $3 \times 3$ strong region yields an error of approximately $4.5 \%$ for the array currents.

The next example corresponds to a thinned array. This array is obtained by randomly throwing away $10 \%$ of the elements of a rectangular 19x19 array. Therefore, when the virtual elements are included, the original 19x19 rectangular array is obtained. Similar to the previous example, Figure 5 shows the comparison of $\left|A_{n m r}\right|$ obtained with the DFT-GFBM and with the conventional hybrid MoM/Green's function technique for the $3^{\text {rd }}, 5^{\text {th }}$ and $9^{\text {th }}$ columns in Figure 5(a), 
Figure 5(b) and Figure 5(c), respectively. Using a $3 \times 3$ strong region with 3 DFT terms yields an error of $5.7 \%$ for the DFT-GFBM results.

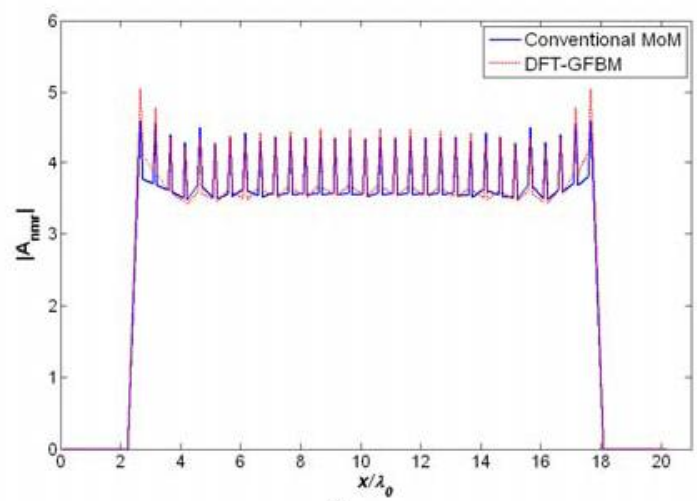

(a) $5^{\text {th }}$ column

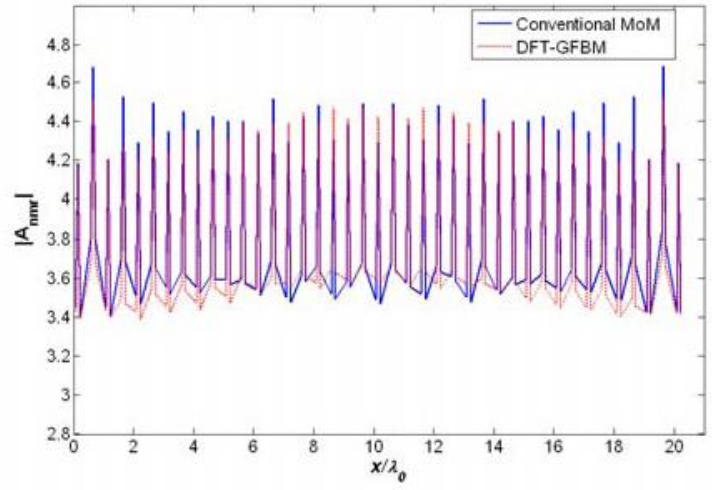

(b) Middle column

Figure 4. Comparison of the magnitude of the induced current $\left|A_{n m r}\right|$ obtained via GFBM/Green's Function-DFT and the conventional MoM/Green's function methods for 1501

element octagonal probe-fed microstrip patch array. Array parameters are: $\mathrm{d}=0.04 \lambda_{0}, \varepsilon_{\mathrm{r}}=2: 55, \mathrm{~L}=\mathrm{W}=0.3 \lambda_{0}$

$\mathrm{dx}=\mathrm{dy}=0.5 \lambda_{0}, \mathrm{xprobe}=-\mathrm{L} / 2 ; \mathrm{yprobe}=0$. Three $\mathrm{x}$-directed basis functions are used for each patch.

As seen in all numerical examples provided in this paper, the agreement between the reference solution and the DFTGFBM solution is fairly good. Accuracy of the solutions can be increased in the expense of increasing the size of the strong region (though we do not recommend it too much) or by slightly increasing the number of DFT terms which might be acceptable for certain applications. More numerical results, particularly for thinned arrays, will be provided during the presentation.

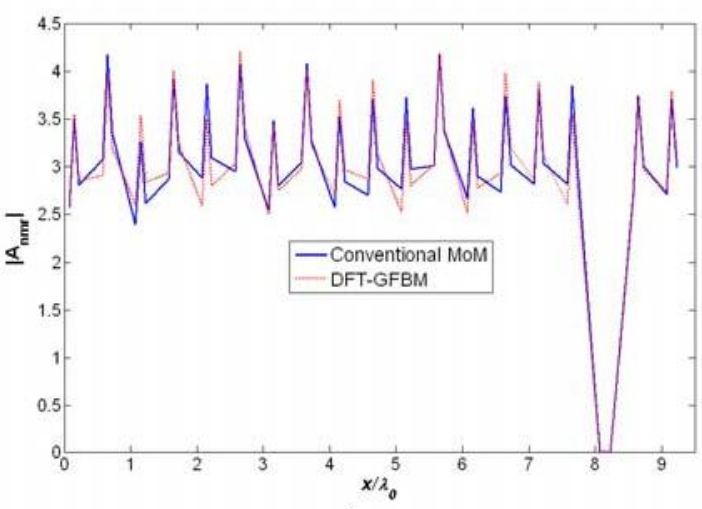

(a) $3^{\text {rd }}$ column

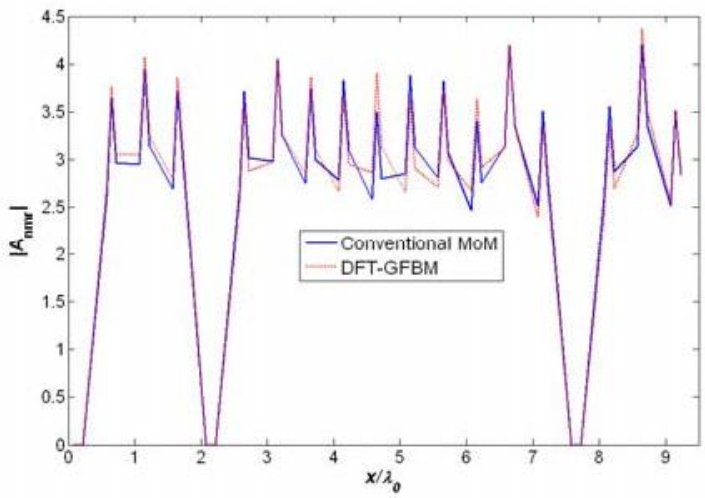

(b) $5^{\text {th }}$ column

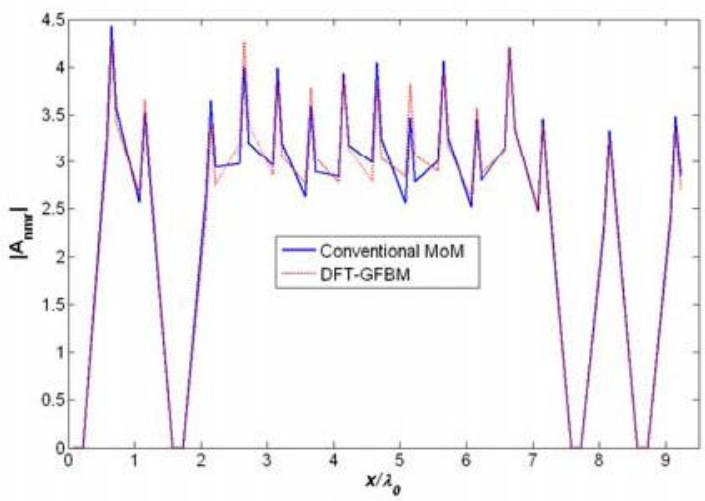

(b) $9^{\text {th }}$ column

Figure 5. Comparison of the magnitude of the induced current $\left|A_{n m r}\right|$ obtained via GFBM/Green's Function-DFT and the conventional MoM/Green's function methods for $10 \%$ randomly thinned 19x19 element probe-fed microstrip patch array. Array parameters are: $d=0.04 \lambda_{0}, \varepsilon_{\mathrm{r}}=2: 55$,

$\mathrm{L}=\mathrm{W}=0.3 \lambda_{0}, \mathrm{dx}=\mathrm{dy}=0.5 \lambda_{0}, \mathrm{xprobe}=-\mathrm{L} / 2 ; \mathrm{y}$ probe $=0$. Three $\mathrm{x}$-directed basis functions are used for each patch. 


\section{Conclusions}

A novel method that can investigate electrically large, arbitrarily contoured and/or thinned finite planar arrays of printed elements with storage and central processing unit (CPU) requirements of $O\left(N_{t o t}\right)$ is developed. With the virtual-element concept, both arbitrarily contoured and/or thinned arrays are first completed to a full rectangular array, where the array elements are identical and the array is periodic. Then a hybrid combination of the Galerkin type MoM and GFBM with the grounded dielectric slab's Green's function is developed and accelerated by a DFT based acceleration algorithm. Numerical results in the form of array current distribution obtained with the proposed method agree well with the solutions obtained via conventional methods.

\section{Acknowled gements}

This project is supported in part by the Turkish Scientific and technical Research Council (TÜBİTAK) under the Grants EEAG-104E044 and Turkish Academy of Sciences (TÜBA)GEBİP

\section{References}

[1] O. Bakır. "Investigation of finite phased arrays of printed antennas on planar and cylindrical grounded dielectric slabs", M.Sc. thesis, Bilkent University Dept. of Electrical and Electronics Engineering, (2006).

[2] O. Bakır, Ö. A. Çivi, V. B. Ertürk, H.-T. Chou. "Efficient analysis of phased arrays of microstrip patches using a hybrid generalized forward backward method/Green's function technique with a DFT based acceleration algorithm", submitted to IEEE Trans. Antennas Propagat.

[3] S. Barkeshli. "An efficient approach for evaluating the planar microstrip Green's function and its applications to the analysis of microstrip antennas and arrays", Ph.D. dissertation, The Ohio-State University Dept. of Electrical Engineering, (1988).

[4] S. Barkeshli, P. H. Pathak, M. Marin. "An asymptotic closed-form microstrip surface Green's function for the efficient moment method analysis of mutual coupling in microstrip antennas", IEEE Trans. Antennas Propagat., 38, pp. 1374-1383, (1989).

[5] H.-C. Chou, H.-K. Ho. "Implementation of a forwardbackward procedure for the fast analysis of electromagnetic radiation/scattering from two dimensional large phased-arrays", IEEE Trans. Antennas Propagat., 52, pp. 388-396, (2004).

[6] Ö. A. Çivi. "Extension of forward-backward method with DFT-based acceleration algorithm for the efficient analysis of radiation/scattering from large finite printed dipole arrays", Microwave and Optical Technology Letters, 37, pp. 20-26, (2003).

[7] Ö. A. Çivi, V. B. Ertürk, H.-T. Chou. "Extension of forward-backward method with DFT-based acceleration algorithm for the efficient analysis of large periodic arrays with arbitrary boundaries", Microwave and Optical Technology Letters, 47, pp. 293-298, (2005).

[8] V. B. Ertürk, H.-T. Chou. "Efficient analysis of large phased arrays using iterative MoM with DFT-based acceleration algorithm", Microwave and Optical Technology Letters, 39, pp. 89-94, (2003).

[9] M. Marin, S. Barkeshli, P. H. Pathak. "Efficient analysis of planar microstrip geometries using a closed-form asymptotic representation of the grounded dielectric slab Green's function", IEEE Trans. Antennas Propagat., 37, pp. 669-679, (1989).

[10] M. Pino, L. Landesa, J. L. Rodriguez, F. Obelliero, R. J. Burkholder. "The generalized forward-backward method for analyzing the scattering from targets on ocean-like rough surfaces", IEEE Trans. Antennas Propagat., 47, pp. 961-968, (1999). 